\title{
Periodic $a b$ initio estimates of the dispersive interaction between molecular nitrogen and a monolayer of hexagonal BN
}

\author{
M. Halo, ${ }^{a}$ S. Casassa,${ }^{a}$ L. Maschio,${ }^{a}$ C. Pisani, ${ }^{a}$ R. Dovesi, ${ }^{a}$ D. Ehinon, ${ }^{b}$ \\ I. Baraille, ${ }^{b}$ M. Rérat ${ }^{b}$ and D. Usvyat ${ }^{c}$ \\ Received 2nd September 2010, Accepted 11th November 2010 \\ DOI: $10.1039 / \mathbf{c 0 c p 0 1 6 8 7 j}$
}

The ab initio determination of the leading long-range term of pairwise additive dispersive interactions, based on the independent analysis of the response properties of the interacting objects, is here considered in the case where these are part of a periodic system. The interaction of a nitrogen molecule with a thin film of hexagonal $\mathrm{BN}$ has been chosen as a case study for identifying some of the problems involved, and for proposing techniques for their solution. In order to validate the results so obtained, the interaction energy between $\mathrm{N}_{2}$ and a $\mathrm{BN}$ monolayer at different distances has been estimated following a totally different approach, namely by performing post-Hartree-Fock (MP2) supercell calculations using the CRYSTAL + CRYSCOR suite of programs. The results obtained with the two approaches closely agree over a long range, while the limit of validity of the purely dispersive regime can be clearly assessed.

\section{Introduction}

In many areas of solid state physics and surface science a lot of attention is being devoted to dispersive interactions. They not only play an essential role in determining the conformation and stability of molecular crystals, but they can contribute a non negligible portion of the cohesive energy of many ionic systems and may control the kinetics of adsorption phenomena. Standard one-electron approximations as are customary in solid-state simulations, such as Hartree-Fock (HF), or Kohn-Sham (KS) formulations of density functional theory (DFT), or hybrid-exchange schemes, are known to be unable to account for them. A number of semi-empirical schemes have been devised therefore in order to estimate $a$ posteriori such interactions. ${ }^{1-5}$ Essentially, after subdividing the whole system into subunits $\left(A_{1}, A_{2}, \ldots\right)$, usually coinciding with the individual atoms, the dispersive energy is evaluated as a sum over all $A_{i}, A_{j}$ pairs of attraction terms of the form $-f(R) C_{n} / R^{n}(n=6,8,10, \ldots) \dagger$ Here $R$ is the distance between the two subunits and $f(R)$ a damping function which prevents the term from acting at close distances, but becomes rapidly a unity with increasing $R$. Both $f(R)$ and the dispersion coefficients $C_{n}$ depend on the "type" of the two interacting

${ }^{a}$ Department of Chemistry IFM and Center of Excellence NIS (Nanostructured Interfaces and Surfaces), Universitá di Torino, via P. Giuria 5, I-10125 Torino, Italy

${ }^{b}$ Equipe de Chimie Physique, IPREM UMR5254, Université de Pau et des Pays de l'Adour, F-64000 Pau, France ${ }^{c}$ Institute for Physical and Theoretical Chemistry, Universität Regensburg, Universitéatsstrasse 31, D-93040 Regensburg, Germany $\dagger$ Another term in $-C_{7} / R^{7}$ due to retardation effect intervenes in the non instantaneous interaction between induced dipole moments. ${ }^{6}$ subunits. The use of such expressions, suitably parameterized, has met with remarkable success in the description of molecular crystals. ${ }^{7}$ Then, in the case of uncharged, apolar interacting subunits, the only important contribution at large distances is given by the van der Waals ( $\mathrm{vdW})-C_{6} / R^{6}$ term. It would be desirable that, at least for this term, $a b$ initio determinations were available for use in solid-state simulations, based on an appropriate partition of the electron distribution and on the a priori estimate of the corresponding vdW $C_{6}$ coefficients.

In the present work we are concerned with one such problem, namely the evaluation of dispersive interactions which take place when a molecule, $M$, interacts with a slab, $\Sigma$, periodic in two dimensions ( $x$ and $y$ ). Two completely different $a b$ initio periodic approaches are adopted. The former obtains the attractive part of the interaction potential based on an independent analysis of the response properties of the interacting systems in terms of their frequency-dependent polarizabilities. These quantities are obtained via a generalization to periodic structures of sum-over-states (SOS) techniques developed in molecular quantum physics ${ }^{8}$ and will therefore be referred to in the following as "SOS". The other approach consists in the explicit evaluation of the total energy of the $M+\Sigma$ system as a function of distance by means of a post-Hartree-Fock periodic technique: the vdW coefficient is here obtained from the analysis of the long-range part of the interaction potential. We use for this purpose our recently implemented CRYSCOR code, ${ }^{9,10}$ which computes the Møller-Plesset perturbative correction at second order (MP2) to the HF solution provided by the CRYSTAL code; ${ }^{11}$ this technique will be designed as "HF+ MP2". 
The special case of a nitrogen molecule interacting with a monolayer of hexagonal boron nitride (h-BN) will be considered here. As already stated in previous work, ${ }^{8}$ the reason for choosing $\mathrm{N}_{2}$ as an adsorbate resides in the importance of this gas for routine quality control and for the characterization of new porous materials. The choice of the substrate is less straightforward: considering the numerous applications of physisorption on activated carbon, a microporous form of graphite, an interesting candidate could have been graphite. However, the semi-metal character of this system prevents its use in both methods: in the SOS technique because the static polarizability component parallel to the slab would result infinite, in the HF + MP2 approach because this method is suitable only for non-conducting systems. Therefore, hexagonal BN, where the different electronegativities of boron and nitrogen result in an experimental band gap of $5.8 \mathrm{eV},{ }^{12}$ seems a reasonable compromise between the interest towards aromatic systems in the context of dispersion and adsorption phenomena and the limitations of our methods. Though not aromatic in the graphene sense, h-BN is isoelectronic to graphite and has the same layered structure, but with different stacking. Besides, h-BN has recently gained interest due to the synthesis of BN nanotubes. ${ }^{13-15}$

When the SOS technique is applied, an additive ansatz will be adopted, namely, we shall assume that each unit cell of $\Sigma$ interacts with $M$ as in an independent-particle model. We also suppose that the slab thickness $t$, the size of the unit cell and that of the molecule are small as compared to the distance $R$ between surface and molecule. The global interaction can then be expressed as a sum of two-body vdW terms involving the general unit cell of the surface and the molecule; the corresponding $C_{6}$ coefficient is obtained by means of the CasimirPolder relation, ${ }^{16}$ which involves the dynamic polarizabilities with respect to an imaginary frequency of the two subunits. While the dynamic polarizability of $M$ can be evaluated using standard techniques of molecular quantum chemistry, the real problem is to have an estimate of the same quantity for each unit cell of the "isolated" surface; in section II, the formulae and algorithms here adopted for this purpose are presented and discussed. They essentially rely on the periodic program CRYSTAL, ${ }^{11}$ which provides the quantum-mechanical description of $\Sigma$ with respect to a variety of one-electron Hamiltonians in a basis set of local Gaussian type functions (GTF) centered in the atoms conventionally designed as atomic orbitals (AO).

In the HF + MP2 approach, the whole system is described as a periodic 2D structure by using a supercell (SC) model of $\Sigma$, and by setting a layer of $\mathrm{N}_{2}$ molecules above it, resulting in one $M$ per SC. CRYSTAL provides the HF solution, while CRYSCOR estimates the MP2 correction to the energy using a local-correlation approach. ${ }^{17-19}$ Note that CRYSCOR is the only quantum-chemistry tool currently available for studying infinite periodic systems in the local-correlation approach. In spite of its limitations, the method presents several distinctive advantages for the present application. First of all, MP2 is known to provide an essentially correct estimate of the correlation correction to the energy, and in particular of dispersive interactions; secondly, its size-consistent character is essential for the application to periodic systems; finally, the local-MP2 variant implemented in CRYSCOR permits the linear scaling of computational costs with respect to the system dimensions. A concise account of the techniques adopted for the present $\mathrm{HF}+\mathrm{MP} 2$ calculations is provided in section III.

The two methods are actually so different that the respective computational parameters are largely independent (see section IV); we have however tried to adjust them so as to obtain similar standards of quality in the two cases.

The results (to be presented in section V) will allow us to analyze the effect of the various computational devices (basis set, truncations, corrections, etc.) adopted in the two cases, but also to appreciate the convergence of the interaction energies over a long range calculated with the two models. From this comparison, the limit of applicability of the purely dispersive regime can also be assessed.

\section{The SOS technique}

When two uncharged, apolar, isotropic and essentially dimensionless systems, $A$ and $B$, are considered, $C_{6}$ can be obtained by means of the Casimir-Polder relation, ${ }^{16}$ which involves the dynamic polarizabilities with respect to an imaginary frequency of the two isolated systems, $\alpha_{A}(\imath \omega)$ and $\alpha_{B}(\imath \omega)$ :

$$
C_{6}=\frac{3}{\pi} \int_{0}^{\infty} \alpha_{A}(\imath \omega) \alpha_{B}(\imath \omega) \mathrm{d} \omega
$$

This formula is easily generalized to the case of non isotropic systems where the tensorial character of the polarizability must be taken into account (see below). It can be shown that this formula is equivalent to the London expression which describes the interaction in terms of induced instantaneous dipoles via second order perturbation theory: ${ }^{20}$

$$
C_{6}=\frac{2}{3} \sum_{i, j} \frac{\vec{\mu}_{A i}^{2} \vec{\mu}_{B j}^{2}}{\Delta E_{A i}+\Delta E_{B j}}
$$

For either system $X(X=A, B), \Delta E_{X i}$ is the excitation energy from the ground to the $i$-th state, and $\vec{\mu}_{X i}=\langle X 0|\vec{r}| X i\rangle$ the corresponding transition moment. In principle, the double sum is extended to all excited states of $A$ and $B$. Here and in the following we assume that in the absence of interaction terms the two systems are in a non-degenerate ground state. The same transition moments and energies appear in the second energy derivative of each system with respect to an electric field with imaginary frequency:

$$
\alpha_{X}(\imath \omega)=\sum_{i} \frac{(2 / 3) \Delta E_{X i} \vec{\mu}_{X i}^{2}}{\Delta E_{X i}^{2}+\omega^{2}} \equiv \sum_{i} \frac{f_{X i}}{\Delta E_{X i}^{2}+\omega^{2}}
$$

where $f_{X i}$ are the so-called oscillator strengths. Eqn (3) can be referred to as the "sum-over-states" (SOS) expression. It is seen that $\alpha_{X}(\imath \omega)$ decreases with $\omega$ and depends essentially on the low-lying excited states of $X$ at small $\omega$. A reasonable description of those states for both isolated systems allows then the value of $C_{6}$ to be estimated via the Casimir-Polder relation.

For atoms and molecules, the expressions just provided can be worked out using accurate post-HF techniques. For those systems, some of us have programmed a time-dependent gauge-invariant (TDGI) method ${ }^{21}$ which is a variational 
perturbation technique taking into account electron correlation effects to describe the low-lying excited states, and in which real or imaginary frequencies can be included. Interaction at long distance between atoms or molecules in their ground (or even excited) state can thus be studied. ${ }^{22}$ Another well-known technique to study intermolecular interactions (including dispersion) is the symmetry-adapted perturbation theory: for the DFT-based versions see ref. 23 and 24.

In order to extend these techniques to the case of interest here, where periodic systems are involved, the critical point of the (accurate) estimation of polarizability for the 2-D periodic substrate needs to be taken into account. In the following this issue is discussed, in particular it is shown how the SOS estimate of the dynamic polarizability of $\Sigma$, based on the use of its one-electron eigenfunctions and eigenvalues (eqn (3)), can be corrected by making reference to its static polarizability calculated independently and more reliably by means of a selfconsistent calculation.

In previous work some of us have programmed the SOS calculation of static and dynamic polarizability of periodic systems ${ }^{25}$ using the approximate solution provided by the code Crystal. ${ }^{11}$ With reference to an effective Hamiltonian $\hat{H}^{\mathrm{eff}}$, the general one-electron eigenstate is a crystalline orbital (CO) which can be labelled by a wavevector index $\vec{k}$ (corresponding to a point of the Brillouin zone and specifying its translational symmetry properties), and by a band index: if the system is an insulator, bands can be classified into occupied, with indices $i, j, k, \ldots$ and virtual ones, with indices $a, b, c, \ldots$ In summary, the general occupied (and virtual) COs and the associated eigenvalues can be written as $|i \vec{k}\rangle \quad(|a \vec{k}\rangle)$ and $\varepsilon_{i \vec{k}}\left(\varepsilon_{a \vec{k}}\right)$, respectively. Within the one-electron model, the ground state is given by the single determinant (detor) constructed with all occupied $\operatorname{COs}\left(\Psi_{0} \equiv\left\|\ldots i \vec{k} \ldots a \overrightarrow{k^{\prime \prime}} \ldots\right\|\right)$, while the excited states are detors obtained by substituting in $\Psi_{0}$ one or more of the occupied COs with virtual ones, that is: $\Psi \ldots a \vec{k}^{\prime \prime} \ldots \equiv\left\|\ldots \vec{i} \ldots \vec{k} \ldots \overrightarrow{k^{\prime \prime}} \ldots\right\| ;$ the corresponding excitation energy is simply given by:

$$
\underset{\ldots E^{\ldots} \vec{k}^{\prime} \ldots}{\stackrel{\vec{k}^{\prime \prime} \ldots}{\ldots}}=\ldots+\varepsilon_{a \vec{k}^{\prime \prime}}+\ldots-\left(\ldots+\varepsilon_{j \vec{k}^{\prime}}+\ldots\right) .
$$

When introducing these results in the SOS expression, eqn (3), advantage can be taken of the fact that the matrix elements of the one-electron dipole operator which defines the transition moments are zero except when the excited state differs from the ground one by just one $\mathrm{CO}$; furthermore, it can be proved that the two COs must belong to the same $\vec{k}$. We then have:

$$
\alpha(\imath \omega)=\sum_{k} w_{k} \sum_{i, a} \frac{f_{i a \vec{k}}}{\Delta E_{i a \vec{k}}^{2}+\omega^{2}}
$$

where $f_{i a \vec{k}}$ are oscillator strengths between occupied $(|i \vec{k}\rangle)$ and virtual $(|a \vec{k}\rangle)$ COs for each $\vec{k}$-point of the Brillouin zone with a geometric weight $w_{k}$, and $\Delta E_{i j \vec{k}}=\varepsilon_{a \vec{k}}-\varepsilon_{i \vec{k}}$ are the corresponding vertical transition energies. In the dipole approximation, valid when the wavelength of the electric field is much larger than the size of the unit cell, the oscillator strength is equal to:

$$
f_{i a \vec{k}}=\frac{2}{3} \Delta E_{i j \vec{k}}\left|\left\langle\overrightarrow{i k}\left|\vec{\Omega}_{k}\right| a \vec{k}\right\rangle\right|^{2}
$$

where $\vec{\Omega}_{k}$ is the field perturbation operator appropriate for periodic systems. ${ }^{26}$ Different expressions can be adopted for $\vec{\Omega}_{k}$. Two of them are considered in the following, to be referred to as the "length" $(\ell)$ or "velocity" ( $v)$ formula, respectively:

$$
\begin{aligned}
\left\langle i \vec{k}\left|\vec{\Omega}_{k}\right| a \vec{k}\right\rangle & =\imath\left\langle i \vec{k}\left|e^{\imath \vec{k} \cdot \vec{r}} \vec{\nabla}_{k} e^{-\imath \vec{k} \cdot \vec{r}}\right| a \vec{k}\right\rangle(\ell) \\
& =\frac{\left\langle\overrightarrow{i k}\left|\vec{\nabla}_{r}\right| a \vec{k}\right\rangle}{\Delta E_{i a \vec{k}}}(v)
\end{aligned}
$$

It has been shown ${ }^{27}$ that the $v$ formula, which is computationally more convenient, is equivalent to the $\ell$ one if the basis set is essentially complete and if the potential part of the Hamiltonian commutes with the position operator (which does not happen with HF or hybrid Hamiltonians containing a non-local exchange term).

This formulation of the SOS technique for calculating the $C_{6}$ coefficient between a molecule and a surface from their respective imaginary frequency polarizabilities has been applied in previous work to describe the adsorption of $\mathrm{N}_{2}$ on the h-BN surface. ${ }^{8}$ In fact, since the molecule and the slab are anisotropic, for each of them two independent polarizabilities must be calculated: $\alpha_{M}^{z z}(\omega)$, and $\alpha_{M}^{x x}(\omega)$ along the molecular axis and perpendicular to it, respectively; $\alpha_{\Sigma}^{z z}(\omega)$, and $\alpha_{\Sigma}^{x x}(\omega)$ normal to the slab and parallel to it, respectively.

However, as just stated, the SOS formula is based on the assumption that the one-electron description of ground and excited eigenstates and eigenvalues of the periodic system is acceptable. It is well known that this is generally not true. In particular, the main gap $E_{\text {gap }}$ between valence and conduction bands in insulators, which defines the lowest excitation energy, largely depends on the one-electron Hamiltonian adopted: with HF it is usually too large by a factor of two or more with respect to the experiment, while it is often underestimated in KS-DFT schemes.

An important check of the reliability of the approximations adopted for the calculation of the frequency-dependent polarizabilities, is based on the comparison of the $\alpha(0)$ value so obtained with the corresponding static polarizability $\underline{\alpha}$. Two techniques have been implemented in CRYSTAL for calculating the latter quantity; ${ }^{11}$ they are both based on the self-consistent evaluation of the second derivative of energy per cell with respect to an applied time-independent electric field. The former consists in the introduction of the static finite field (FF) perturbation in the SCF process as a "sawtooth electric potential", ${ }^{28}$ which allows the system to be treated as periodic by using a supercell in the direction of the field $;{ }^{29,30}$ precisely because of this supercell trick, calculations are rather lengthy. More accurate and less time-consuming results can be obtained using the analytical coupled-perturbed HF or KS (CPHF or CPKS) method for periodic systems, recently implemented and validated. ${ }^{31,32}$ In the following, only CPKS will be considered since DFT Hamiltonians are used to describe the surface. With respect to the SOS method, the response of the system to the external perturbation is here taken into account: interestingly, the SOS estimate of the static polarizability turns out to be the zero step of the iterative CPKS process. 
In the case of nanotubes, Benedict et al. ${ }^{33}$ have shown that the transverse polarizability value calculated when orbital relaxation effects are taken into account as in CPKS (or in the finite field FF method) is very different from the unrelaxed (SOS) one. The authors show that this important relaxation effect can be modeled by the Clausius-Mossotti formula relating the SOS and CPKS polarizability values with the depolarization factor equal to $2 \pi$ when the field is transverse to the tube. ${ }^{34}$ On the contrary, when the field is parallel to the cylinder, the effect is much weaker leading to SOS and CPKS values very similar to each other, as has also been found by Brothers et al. $^{35}$ (the depolarization factor is zero in that direction of the field).

In this work, where the 2-D BN system is studied, the relaxation effect has also been found to be very large when the field direction is perpendicular to the slab, while SOS and CPKS values of the parallel component of the polarizability are similar. Then, the use of SOS for calculating $\alpha_{\Sigma}^{x x}(l \omega)$ is well justified provided that the gap is well described. This is not true with $\alpha_{\Sigma}^{z z}(\omega)$, where the depolarization factor due to the medium is $4 \pi,{ }^{34}$ leading to an important relaxation effect of the crystalline orbitals. Corrections to the SOS method are needed in this case. Following the model of Benedict et al., ${ }^{33}$ we have used the Clausius-Mossotti relation with the depolarization factor equal to $4 \pi$ (2-D system) instead of $2 \pi$ (1-D) in order to retrieve the "corrected" value of the normal polarizability of the slab from the SOS estimate:

$$
\alpha_{\Sigma}^{z z}(\imath \omega ; \text { corr })=\frac{\alpha_{\Sigma}^{z z}(\imath \omega ; \text { SOS })}{1+4 \pi \alpha_{\Sigma}^{z z}(\imath \omega ; \text { SOS }) / V}
$$

However, the volume $V$ is not defined for the slab, and we have to determine it independently. For this purpose, the $\alpha_{\Sigma}^{z z}$ (0; corr) value in the previous equation is fitted to the static $\underline{\alpha}_{\Sigma}^{z z}[\mathrm{CPKS}]$ value: it turns out that the region in which the electron cloud is polarized corresponds to assigning to each unit cell a thickness approximately equal to the $c$-parameter of h-BN bulk.

In summary, the following sequence of steps is adopted in order to estimate the molecule-slab vdW interaction:

1. Select a suitable one-electron KS Hamiltonian and calculate excitation energies and oscillator strengths for the slab.

2. Calculate the corresponding $\alpha_{\Sigma}^{z z}(l \omega ;$ SOS $)$ and $\alpha_{\Sigma}^{x x}(\imath \omega ;$ SOS $)$ using the SOS formula.

3. Calculate the static polarizability $\underline{\alpha}_{\Sigma}^{z z}[\mathrm{CPKS}]$ of the slab in the normal direction by means of the CPKS technique using either the $\ell$ or the $v$ formula (eqn (6)).

4. Determine the effective volume $V$ in eqn (7) by imposing the equality: $\alpha_{\Sigma}^{z z}(0 ;$ corr $)=\underline{\alpha}_{\Sigma}^{z z}[\mathrm{CPKS}]$.

5. Calculate $\alpha_{M}^{z z}(\imath \omega ;$ TDGI $)$ and $\alpha_{M}^{x x}(\imath \omega ;$ TDGI $)$ using the TDGI method.

6. Obtain the four mixed $C_{6}^{U T}$ values (with $U, T=z z, x x$, and the first two indices referring to the molecule, the last two to the slab): see section IV-B for details.

7. For a given distance $R$ between the molecule and the surface, integrate the $C_{6} /\left(R^{2}+r^{2}\right)^{3}$ terms over the $r$ component parallel to the surface, resulting in an interaction energy $E(R)=-C_{4} / R^{4}$, with a $C_{4}$ coefficient depending on the orientation of the molecule, either perpendicular $(\perp)$ or parallel $(\|)$ to the surface ( $A$ being the area of the 2-D unit cell):

$$
\begin{aligned}
& C_{4}^{\perp}=\frac{3 \pi}{4 A}\left(C_{6}^{x x x x}+C_{6}^{x x z z}+C_{6}^{z z x x}+C_{6}^{z z z z}\right) \\
& C_{4}^{\|}=\frac{3 \pi}{8 A}\left(C_{6}^{x x x x}+C_{6}^{x x z z}+3 C_{6}^{z z x x}+3 C_{6}^{z z z z}\right)
\end{aligned}
$$

In addition to the case of the $\mathrm{BN}$ monolayer, some data on the static polarizability of $\mathrm{BN}$ slabs of different thickness and on $\mathrm{BN}$ bulk have also been obtained in order to get more insight into the influence of computational parameters, and will be reported in section $\mathrm{V}$. The dispersion coefficients concerning the interaction of the $\mathrm{BN}$ slabs with the nitrogen molecule have also been estimated with the technique just described, by supposing again that the slab thickness is very small as compared to the distance between the molecule and the surface.

\section{HF + MP2 estimate of interaction energies}

The HF + MP2 approach implemented in the CRYSTAL + CRYSCOR suite of programs and adopted in the present work provides both the attractive and the repulsive contribution to the interaction energy and is valid for all adsorbate-substrate distances. In fact, the system is here considered as a whole, and treated as a periodic 2-D structure. For this reason, SC calculations are needed in order to avoid intermolecular interactions which are absent in the SOS model. It can be noted incidentally that the MP2 estimate of the interaction energy provided by CRYSCOR is conceptually similar to that implied in London's formula, eqn (2), since in both cases use is made of second order perturbation theory. However, in the former case reference is made to the exact ground state of the two non interacting systems while, in the latter, the reference state is the HF wavefunction of the compound system. Besides, the MP2 method treats dispersion at the uncoupled$\mathrm{HF}$ level, since orbital relaxation effects are not present in the formalism. This can lead in some cases to noticeable overestimation of dispersion, especially pronounced for $\pi-\pi$ interaction. Another basic difference is due to the fact that in the SOS model only the orientation of the molecule with respect to the surface needs to be specified, whereas we must provide in our case the exact relative position of the two subsystems. The case of the molecule oriented perpendicular to the $\mathrm{BN}$ monolayer and pointing towards the center of the hexagon (which has been recognized as the preferred adsorption site for non-polar molecules ${ }^{36}$ ) is the only one to be considered in the following.

The parameters used for the present CRYSTAL + CRYSCOR calculations will be reported in section IV; here we just recall the main concepts and quantities involved in the local-MP2 (LMP2) approach adopted in CRYSCOR, ${ }^{9,10}$ as are needed to follow the discussion in the next sections.

The basic ingredients of the LMP2 approach are (i) Wannier functions (WFs, labelled $i, j, \ldots$ ), a set of well-localized, symmetry-adapted, mutually orthogonal, translationally equivalent functions, which span altogether the valence $\mathrm{HF}$ manifold and are provided by the CRYSTAL code and (ii), projected atomic orbitals (PAOs, labelled $a, b, \ldots$ ), a set of 
non-orthogonal functions spanning the virtual space, obtained in CRYSCOR from the individual AOs of the basis set by projecting out their component in the occupied HF manifold.

To each WF a domain is associated, consisting in a certain number of atoms whose AOs contribute significantly to its Mulliken population: for each pair of WFs $(i, j)$ involved in a biexcitation, a pair-domain $\mathscr{D}_{(i j)}$ is then defined which is simply the union of the corresponding domains. Owing to the locality ansatz, only those $[(i j) \uparrow \uparrow(a b)]$ biexcitations for which both PAOs $a$ and $b$ belong to atoms in $\mathscr{D}_{(i j)}$ are retained.

The second consequence of the locality ansatz is that the distance between the centers of the two WFs involved in retained biexcitations can be imposed to be less than a given cut-off radius $D$. Note that, due to translational periodicity, it can always be assumed that the first WF in the pair is located in the reference zero cell.

A third computational parameter must be defined in order to get rid of infinite summations: since each WF (PAO) is expressed as a linear combination of AOs, $\phi_{i}=\sum_{\mu} c_{\mu i} \chi_{\mu}$, sums over AOs extend in principle to the whole periodic system. A tail-truncation parameter $t$, allows us to truncate these sums, by setting to zero those coefficients for which $\left|c_{\mu i}\right|<t$.

An important advantage of the local approach is the possibility to select a subset of biexcitations according to the WFs (and as a consequence the PAOs) involved. In the present case, we actually have two separate subsystems, the molecule and the slab, and are interested in the correlation interactions between rather than inside them. Therefore, the biexcitations, for which one WF (and the respective domain) belongs to the slab, the other (with its domain) to the molecule, are representing the dispersion ${ }^{37}$ and are the most important for our purposes. However, the intramolecular and intra-slab biexcitations cannot simply be neglected, since this would lead to significant overestimation of the interaction energy, especially at short molecule-surface distances. Indeed, when the molecule and the slab come close to each other, the respective electron distributions are modified in order to minimize the Pauli repulsion, which makes the densities of the interacting monomers more "compact" relative to the free ones. In the compact densities the electrons possess less freedom to avoid each other, and the intramolecular and intra-slab correlation energies go up (or decrease in the absolute value). This repulsive contribution to the interaction energy is not big, but on the scale of the weak interactions can be noticeable and should thus be evaluated. At the same time, the convergence of the intramolecular and intra-slab contribution to the correlation energy is relatively fast, and in most cases it is sufficient to include only close-by intra-pairs. Such a technique allows for considerable savings in the computational resources and is used throughout our LMP2 study.

The slab-molecule interaction energy at a given distance $R$ will be finally evaluated by subtracting from the total energy at that distance the one obtained with the same computational settings at a very large distance $\left(R_{\infty}\right)$. The result so obtained is corrected for the basis set superposition error (BSSE), following the standard counterpoise (CP) technique. ${ }^{38}$ As is shown in section V-B, the BSSE correction is small in all cases, but more important for the HF than for the MP2 contribution and becomes smaller, as expected, with increasing $R$ and with improvement of the BS.

\section{Computational settings}

\section{A. General issues}

Since our final aim is to compare SOS and HF + MP2 results for the long-range portion of the interaction curve, we have tried to adopt as far as possible the same computational settings in the two cases, which is a non trivial task due to the fundamental diversity of the two methods. Here we report on some general computational issues, especially as concerns the use of CRYSTAL, leaving the more specific aspects to the following subsections.

Geometry. For both subsystems, the geometry was frozen at the experimental values, that is: $a=2.504 \AA$ for the h-BN monolayer, and $l=1.0943 \AA$ for the triple bond in the $\mathrm{N}_{2}$ molecule. For the HF + MP2 calculations a $4 \times 4$ SC was used resulting in a distance of $10.016 \AA$ between neighbouring molecules. The corresponding intermolecular interaction is very small, and remains constant while varying the molecule/ slab distance, so it cancels out when performing differences. As a matter of fact, many of the calculations were repeated with a $2 \times 2 \mathrm{SC}$ and were seen to provide very similar results.

Hamiltonians. The CRYSTAL calculations providing information on the BN system as needed for the SOS approach were performed using the one-electron BLYP Hamiltonian. ${ }^{39,40}$ There are two main reasons for this choice: (i) the $v$ formula for the calculation of the static polarizability (see eqn (6)) requires a local exchange-correlation potential to be used; (ii) among the KS-DFT Hamiltonians which satisfy this requirement, BLYP was found to provide a reasonable estimate of the fundamental gap (see section IV-B).

In the other approach, the use of the HF solution from CRYSTAL is mandatory for evaluating in CRYSCOR the MP2 correction.

Basis sets. The choice of the GTF-AO basis set (BS) is a delicate issue and has different aspects for the two approaches. In SOS, the two systems are independent, while in the $\mathrm{HF}+\mathrm{MP} 2$ approach we must describe the composite system; furthermore, the MP2 calculation requires a better description of the virtual HF manifold than is the case with the other technique. The BSs tested in this study will be indicated with the notation $X / Y / Z$, where $X$ and $Y$ refer, respectively, to the $\mathrm{BS}$ of the $\mathrm{B}$ and $\mathrm{N}$ atoms in the slab and $\mathrm{Z}$ to that of molecular nitrogen, and will be assigned a conventional name.

BSA and BSB will design two BSs to be used for both techniques. The former, 6-31G*/7-31G*/cc-pVTZ, adopts double-zeta plus polarization (pDZ) sets for the slab atoms as used and partially reoptimized in previous work, ${ }^{8}$ while for the molecular atoms a correlation-consistent (cc) triple-zeta (TZ) quality set taken from Dunning is used; ${ }^{41}$ in the latter, Hess(d)/Hess(d)/cc-pVTZ, a richer set is used for the slab atoms, which is of pVTZ type, and was proposed by Hess specifically for bulk h-BN, providing solutions close to the HF limit. ${ }^{42}$ In addition, a number of other BSs have been tried in the $\mathrm{HF}+\mathrm{MP} 2$ case, to be described in section IV-C. 
Truncation tolerances in CRYSTAL. The TOLINTEg parameters which control the truncation of the Coulomb and exchange lattice series in CRYSTAL $^{11}$ have been set to very tight values $(8,8,8,16,32)$ in order to achieve convergence with the BSs adopted. For the rest, the standard default settings are used.

\section{B. Details on the SOS technique}

The polarizabilities of the isolated $\mathrm{N}_{2}$ molecule have been obtained at the configuration interaction level of theory, ${ }^{43,44}$ using the TDGI method ${ }^{21}$ with a specific triple-zeta BS proposed by Sadlej. ${ }^{45-47}$ Computational details can be found in the previous work of Baraille et al. ${ }^{8}$

The issue of the KS-DFT Hamiltonian to be adopted for the calculation of the polarizability of $\Sigma$ is now briefly addressed. Reference is made to the properties of bulk h-BN, as no experimental information is available in this respect, to our knowledge, for the thin films we are here interested in. The subject has recently been reviewed by Museur et al. in a study of the defect-related photoluminescence of h-BN (see ref. 48 and references therein). Without going into details, the analysis of the experimental data performed in that paper supports the results obtained theoretically by Arnaud et al. using an all-electron GW approximation, ${ }^{49}$ and predicting an indirect bandgap of $5.95 \mathrm{eV}$ between the bottom of the conduction band at the $M$ point and the top of the valence band near the $\mathrm{K}$ point, and a lowest direct interband transition of $6.47 \mathrm{eV}$ located at the $\mathrm{H}$ point. The BLYP method provides qualitative agreement with that band structure, although the gaps are underestimated, as is customary with DFT approximations: with BSB, it gives an indirect gap of $4.48 \mathrm{eV}$ and a direct gap at $\mathrm{H}$ of $4.65 \mathrm{eV}$. Quite similar results are obtained with BSA. These values are larger than those obtained with local-density DFT approximations by about $5 \%$, but still below their best estimates by almost $30 \%$. This may result into too large calculated polarizabilities: note however that the error should be much smaller than that figure, because bandwidths (both in the virtual and in the occupied manifold) are reasonably well described. As expected, the $\mathrm{BSB} / \mathrm{HF}$ interband transitions are largely overestimated with an indirect gap of $13.58 \mathrm{eV}$ and the lowest direct vertical transition energy of $14.03 \mathrm{eV}$. A hybridexchange Hamiltonian would probably be a better choice than BLYP but, as stated above, this is not feasible with our tools presently available.

For the CPKS determination of the static polarizability of the slab, we have used both the $\ell$ and $v$ techniques (eqn (6)), but only the latter one in the SOS expressions providing the dependence of polarizabilities from imaginary frequencies (eqn (4)).

After applying the correction of eqn (7) to the normal polarizability of the slab, two methods are used to evaluate the $C_{6}$ coefficients (step 6 in the procedure outlined in section II). The first one (truncated integration, or briefly "int") is the numerical integration of the product of the dynamic polarizabilities, $\Pi_{M \Sigma}^{U T}(\omega) \equiv \alpha_{M}^{U}(\omega) \alpha_{\Sigma}^{T}(\omega)$, following eqn (1) up to $\omega=2$ a.u. Since the product decreases quite slowly with respect to $\omega$, the $C_{6}$ value obtained is underestimated. The second technique ("fit") consists in fitting $\Pi_{M \Sigma}^{U T}(\omega)$, in the explored $\omega$ range, with a parametric expression of the form:

$$
\left[\sum_{i=1}^{3} \frac{2 e_{M i}\left(m_{M i}^{U}\right)^{2}}{e_{M i}^{2}+\omega^{2}}\right] \times\left[\sum_{j=1}^{3} \frac{2 e_{\Sigma j}\left(m_{\Sigma j}^{T}\right)^{2}}{e_{\Sigma j}^{2}+\omega^{2}}\right]
$$

The best fit parameters $\left\{e_{M i}, m_{M i}^{U}\right\},\left\{e_{\Sigma j}, m_{\Sigma j}^{T}\right\}$ are then interpreted, by comparison with eqn (3), as "effective" transition energies and moments and used for obtaining the vdW coefficients owing to a London-like expression (see eqn (2) for the mean value):

$$
C_{6}^{U T, \mathrm{fit}}=6 \sum_{i, j} \frac{\left(m_{M i}^{U}\right)^{2}\left(m_{\Sigma j}^{T}\right)^{2}}{e_{M i}+e_{\Sigma j}}
$$

The values so obtained are slightly larger (and more reliable, in our opinion) than with the other technique.

\section{Details on the HF + MP2 technique}

As mentioned in section III, in order to gain in efficiency we use different pair cut-off distances for the inter- and intra-system pairs, the latter being considerably reduced. The contribution to the interaction energy from the inter-system excitations within a cut-off radius of $15 \AA$ was explicitly accounted for; for those beyond, a "Lennard-Jones" extrapolation was used ${ }^{9,10}$ which however turned out to be almost negligible and nearly independent of the distance. Within each subsystem, instead, only biexcitations up to a distance of $3 \AA$ are retained: this corresponds to including all excitations within the molecule, but only short-range ones within the slab with an important reduction of computational cost.

The domains of the WFs of the slab, which are essentially centered on nitrogens, consist of 10 atoms, the reference $\mathrm{N}$ atom plus its $3 \mathrm{~B}$ and $6 \mathrm{~N}$ first neighbors; for WFs in the molecule, they consist of both molecular atoms. The cut-off threshold $t$ for WF and PAO tails is set to the tight 0.0001 value. The 2-electron repulsion integrals (ERIs) are evaluated by means of the density fitting technique extended to periodic systems ${ }^{50}$ up to a distance of $5 \AA$ between the involved WF, and with a multipolar technique beyond.

The domain size and pair-lists for the density-fitting- and multipole-expansion-treated integrals once obtained with the above parameters within the $\mathrm{N}_{2}$-slab distance of $2.5 \AA$ have been fixed and used further without modification for all the considered geometries. Besides, in order to avoid bumps in the potential curves the "freezing of indices" procedure has been also applied for the HF calculations (where the number of integrals was kept constant) and WF localization and symmetrization (where the WFs of the previous geometry were used as the starting guess for those of the next geometry, thus maintaining their order and symmetry classification).

A careful study of the influence on MP2 interaction energies of the BS assigned to the atoms in the slab has been carried out by improving their description beyond the level provided by the high quality BSB (see section IV-A). In particular, a further series of $d$ and $f$ functions has been added to BSB, giving rise to BSC [or Hess(dd)/Hess(dd)/cc-pVTZ], which contains two $d$ single-Gaussian GTFs with exponents $\alpha_{d}^{\prime}=1.2, \alpha_{d}^{\prime \prime}=0.4$ a.u. (instead of the original $d$ with exponent 
$\alpha_{d}=0.8$ a.u.) and BSD [or Hess(ddf)/Hess(ddf)/cc-pVTZ], containing in addition an f-type GTF with exponent $\alpha_{f}=$ 1.4 a.u. An additional series of augmented BSs has also been tried for a $2 \times 2 \mathrm{SC}$ slab, which is far less costly from a computational point of view; these correspond to an aug-ccpVTZ basis for the molecule, as proposed by Dunning, ${ }^{41}$ and a partially augmented BS for the slab, where only the $d$ and $f$ augmented functions ${ }^{51}$ of the standard aug-cc-pVTZ are added to the previously listed bases, by means of the dual basis set technique. ${ }^{52}$ The performance of these bases is discussed in section V-B.

\section{Results and discussion}

\section{A. Van der Waals coefficients in the SOS approach}

Tables 1 and 2 report values of the static polarizability parallel and perpendicular to the $\mathrm{BN}$ layers, for slabs of different thickness and for bulk BN. The results provide indications on the adequacy of the computational techniques adopted. The following can be noted.

The $\underline{\alpha}^{x x}$ values reported in Table 1 are similar in all cases ( $\approx 35 a_{0}^{3}$ per formula unit). Convergence with increasing slab thickness to the bulk result is rapidly reached. The SOS and CPKS estimates are also very close to each other. As concerns the use of the $\ell$ or $v$ transition moment expression (eqn (6)) in the oscillator strength (eqn (5)), and then in the polarizabilty (eqn (4)), which should coincide with complete BSs, it is seen that the results are in closer agreement with the basis set BSB than BSA for the parallel component of the polarizability. The relative difference between the length and velocity values is less than $10 \%$ with BSB and more than $20 \%$ with BSA.

The $\underline{\alpha}^{z z}$ values in Table 2 present two striking differences: the very large discrepancy between the SOS and CPKS estimates, and the non-convergence of the CPKS results to the bulk value with increasing slab thickness. The former effect has already been commented on when introducing eqn (7); it is worthwhile to briefly discuss the latter.

Polarizability is the second derivative of the energy with respect to the displacement (external) field for the slabs, and to the macroscopic (internal) field for the bulk. These fields are essentially equal in the parallel direction, but not in the $z$ one, along which the slabs are finite (the operator $\Omega_{k}$ reduces in this case to the usual operator $r$ ), and relaxation effects are important. These effects are correctly taken into account by

Table 1 Parallel component of the static polarizability, $\underline{\alpha}^{x x}$, per BN unit formula (in $a_{0}{ }^{3}$ ), for several slabs $\mathrm{S} n$ ( $n$ indicating the number of layers in the slab) and for bulk BN. Different computational conditions have been used, as indicated: basis set (BSA or BSB); SOS or CPKS technique, with the $\ell$ or $v$ expression for the $\vec{\Omega}_{k}$ operator (eqn (6))

\begin{tabular}{lllllll}
\hline Basis set & Technique & S1 & S2 & S5 & S13 & Bulk \\
\hline BSA & SOS $(v)$ & 31.32 & 31.28 & 31.20 & 31.17 & 31.15 \\
& CPKS $(v)$ & 30.69 & 30.64 & 30.57 & 30.54 & 30.53 \\
& SOS $(\ell)$ & 38.70 & 37.80 & 37.27 & 37.04 & 36.90 \\
BSB & CPKS $(\ell)$ & 37.88 & 37.00 & 36.47 & 36.21 & 36.12 \\
& SOS $(v)$ & 32.63 & 32.52 & 32.45 & 32.42 & 32.40 \\
& CPKS $(v)$ & 32.03 & 31.93 & 31.86 & 31.83 & 31.81 \\
& SOS $(\ell)$ & 35.14 & 34.92 & 34.78 & 34.73 & 34.70 \\
& CPKS $(\ell)$ & 35.14 & 34.20 & 34.08 & 34.03 & 34.00 \\
\hline
\end{tabular}

the CPKS technique. As a matter of fact, the ratio between the BSB/CPKS $\underline{\alpha}^{z z}$ estimates for the bulk and S13 (13.37/5.58 = 2.40) is similar to the perpendicular component of the dielectric constant of the bulk (the experimental highfrequency value of $\varepsilon^{z z}$, as provided by Rumyantsev et al., ${ }^{53}$ is 2.2), which is precisely the ratio between the displacement and the macroscopic field. This value can be retrieved by the formula relating the dielectric constant to the polarizability of bulk h-BN in the $c(z)$ direction: $\varepsilon^{z z}=1+4 \pi \underline{\mathrm{buulk}}_{\mathrm{z}}^{z z} / V=2.38$, the volume being equal to $V=122 \mathrm{bohr}^{3}$, or the polarizability of the slab but with the relation: $\varepsilon^{z z}=1 /\left(1-4 \pi \underline{\alpha}_{S 13}^{z z} / V\right)=$ 2.35 (see reference CPHF). Not surprisingly, the SOS technique, which ignores relaxation effects, provides essentially the same $\underline{\alpha}^{z z}$ values for the thick slabs and the bulk.

Fig. 1 reports the polarizabilities of the $\mathrm{N}_{2}$ molecule and of the $\mathrm{BN}$ monolayer as a function of the imaginary frequency, as obtained with the different techniques here described. From these functions, the $C_{6}$ values for the four possible relative orientations have been obtained using one of the two techniques, "int" or "fit", described in section IV-B.

After substitution in eqn (8), we finally obtain the $C_{4}$ values reported in Table 3, relative to the interaction of the molecule, in either of the two orientations, with $\mathrm{S}_{1}$ or $\mathrm{S}_{2}$. It can be noticed that the large differences between the SOS and CPKS estimates of the perpendicular polarizability of the slab (see Table 2) are smoothed out after performing the integration.

\section{B. Interaction energies: critical comparison between the SOS and HF + MP2 methods}

Fig. 2 compares the $M / \Sigma$ interaction energies between the BN monolayer and a nitrogen molecule perpendicularly adsorbed above it, obtained with the two approaches. All HF and $\mathrm{HF}+\mathrm{MP} 2$ data here reported are corrected for BSSE (see below), and they correspond to adsorption above the center of the hexagon, at a distance $R$ measured from the surface plane to the center of the molecule. For the $-C_{4}^{\perp} / R^{4}$ curve, our "best" estimate of the $\mathrm{vdW}$ coefficient has been used, namely 7.01 a.u. (see Table 3). Let us comment on these data.

The HF curve is seen to be repulsive at all distances; the BSB data are reported here, but very similar results are obtained with all BSs. All $\mathrm{HF}+\mathrm{MP} 2$ curves present instead a clear minimum at $R \approx 4.0 \AA$, corresponding to a $\mathrm{N}$-surface distance of $\approx 3.5 \AA$. Results for BSC are not reported in the plot for the sake of clarity but they are in between BSB and BSD ones. The "physisorption-like" behaviour of these curves is noteworthy: a very steep repulsive regime due to the Pauli repulsions described by the HF term, is followed by the comparatively much smoother regime beyond the minimum, where dispersive forces are dominating.

The adsorption energy $E_{\text {ad }}$ increases with increasing BS quality, namely from 1.7 to $2.1 \mathrm{mE}_{h}$ while passing from BSA to BSD.

As already mentioned, a series of augmented BS has been tried in the $2 \times 2 \mathrm{SC}$ case, far less costly from a computational point of view, the results of which are in great part transferable to the $4 \times 4 \mathrm{SC}$, as shown by the proximity of the two curves for BSB in Fig. 2 (but similar results are obtained for the other 
Table 2 Perpendicular component of the static polarizability, $\underline{\alpha}^{z z}$, in the same units, for the cases, and with the computational techniques as in Table 1

\begin{tabular}{lllllll}
\hline Basis set & Technique & S1 & S2 & S5 & S13 & Bulk \\
\hline BSA & SOS $(v)$ & 8.57 & 10.34 & 11.57 & 12.07 & 12.39 \\
& CPKS $(v)$ & 3.70 & 4.14 & 4.40 & 4.51 & 10.07 \\
& SOS $(\ell)$ & 10.35 & 12.63 & 14.22 & 14.86 & 15.27 \\
BSB & CPKS $(\ell)$ & 4.23 & 4.84 & 5.21 & 5.36 & 12.45 \\
& SOS $(v)$ & 9.21 & 10.96 & 12.14 & 12.63 & 12.94 \\
& CPKS $(v)$ & 3.73 & 4.19 & 4.47 & 4.59 & 10.91 \\
& SOS $(\ell)$ & 12.56 & 14.16 & 15.23 & 15.67 & 15.94 \\
& CPKS $(\ell)$ & 5.07 & 5.35 & 5.51 & 5.58 & 13.37 \\
\hline
\end{tabular}
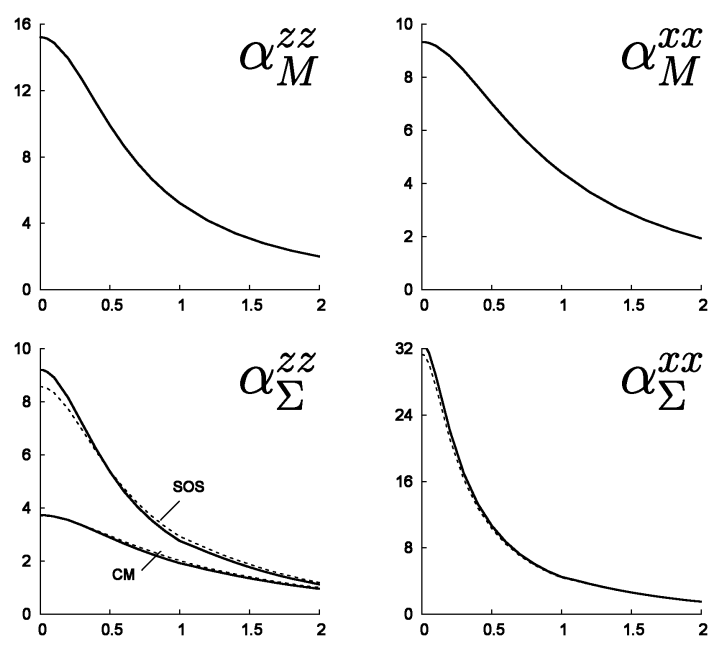

Fig. 1 Polarizability of the $\mathrm{N}_{2}$ molecule (top panels) and of the $\mathrm{BN}$ monolayer (bottom panels) as a function of the imaginary frequency, both expressed in a.u. On the left, the direction is that of the molecular axis and of the normal to the slab, respectively, conventionally indicated as $z$; on the right, the perpendicular one $(x)$. In the $\mathrm{BN}$ plots, the dashed and continuous curves refer to the use of BSA or BSB. In the bottom left plot, the $\alpha_{\Sigma}^{z z}(\imath \omega ; \mathrm{SOS})$ functions and the Clausius-Mossotti correction (CM, eqn (7)) are both reported.

Table 3 Van der Waals $C_{4}$ coefficients (a.u) for $\mathrm{S}_{1}$ and $\mathrm{S}_{2}$ (the latter divided by two, to make comparisons easier), computed with two BSs, and using the int or fit techniques. In all cases, (SOS) indicates that the $\alpha_{\Sigma}^{z z}(\omega ; \mathrm{SOS})$ function has been used for the slab, while (corr) indicates that $\alpha^{z z}(\omega$;corr) has been used instead, owing to eqn (7)

\begin{tabular}{|c|c|c|c|c|c|}
\hline \multirow[b]{2}{*}{ Basis set } & \multirow[b]{2}{*}{ Technique } & \multicolumn{2}{|l|}{$\underline{S_{1}}$} & \multicolumn{2}{|l|}{$\underline{\mathrm{S}_{2}}$} \\
\hline & & $C_{4}^{\perp}$ & $C_{4}^{\|}$ & $C_{4}^{\perp}$ & $C_{4}^{\|}$ \\
\hline \multirow[t]{4}{*}{ BSA } & int (SOS) & 7.17 & 6.43 & 7.43 & 6.67 \\
\hline & fit (SOS) & 7.91 & 7.16 & 8.19 & 7.41 \\
\hline & int (corr) & 6.15 & 5.52 & 6.25 & 5.61 \\
\hline & fit (corr) & 6.82 & 6.18 & 6.94 & 6.28 \\
\hline \multirow[t]{4}{*}{ BSB } & int (SOS) & 7.44 & 6.67 & 7.66 & 6.88 \\
\hline & fit (SOS) & 8.20 & 7.45 & 8.40 & 7.60 \\
\hline & int (corr) & 6.35 & 5.70 & 6.44 & 5.78 \\
\hline & fit (corr) & 7.01 & 6.35 & 7.11 & 6.44 \\
\hline
\end{tabular}

bases as well). A significant increase of the interaction energy is seen around the minimum, which rises up to $3.3 \mathrm{mE}_{h}$ and undergoes a corresponding shift towards closer distances between molecule and slab, at $\approx 3.8 \AA$ (corresponding to a $\mathrm{N}$-surface distance of $\approx 3.3 \AA$ ). This shows that we have not

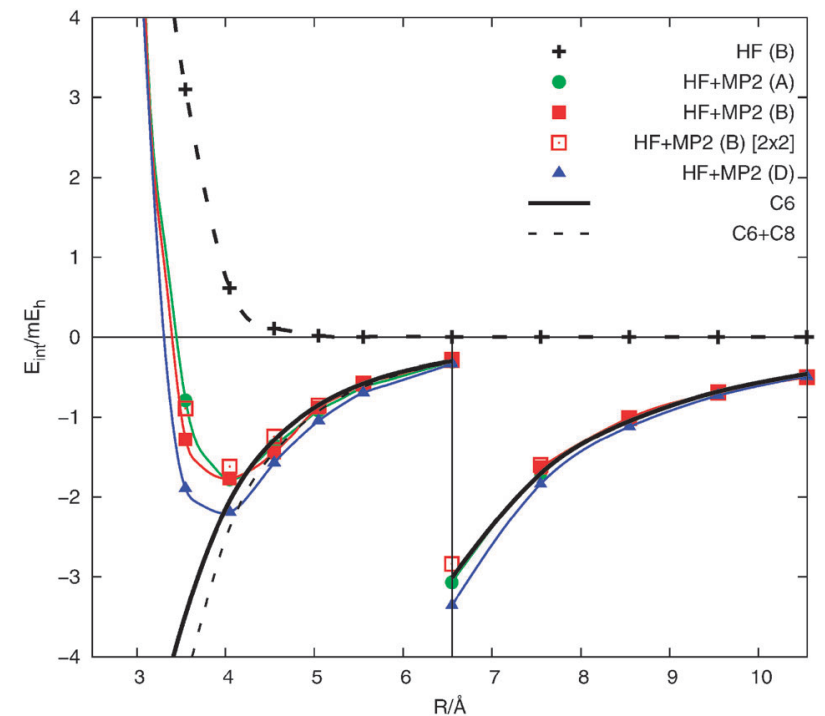

Fig. 2 Interaction energy between nitrogen and the $\mathrm{BN}$ monolayer at different levels of theory: HF/BSB (crosses); HF + MP2/BSA (circles), /BSB (squares), /BSD (triangles); the $\mathrm{HF}+\mathrm{MP} 2 / \mathrm{BSC}$ data are not reported, but they are intermediate between the BSB and BSD ones. The HF + MP2/BSB data for the $2 \times 2 \mathrm{SC}$ (open squares) are also reported for the sake of comparison. The thin curves are interpolations between the calculated points; the thick curve $\left(-\mathrm{C}_{4}^{\perp} / R^{4}\right)$ is the $C_{6}$ estimate of the vdW interaction energy. Beyond $R=6.5 \AA$, all data have been multiplied by a factor of 10 : in this region, most results for the various BSs are overlapping on the scale of the plot. The dashed curve is the estimated " $C_{6}+C_{8}$ " interaction energy (see text for details).

yet reached the MP2 basis completeness; a more complete study would be required in order to fully understand the effects of augmented function on the short-range portion of the interaction energy curve, which is outside the scope of the present paper and will be matter of future work.

The adsorption energy value we obtain with our best basis set seems in reasonable agreement with literature data concerning similar systems. Curthoys and Elkington, ${ }^{36}$ using semi-empirical values for the atom-atom vdW coefficients, estimated that argon (the polarizability of which is very close to that of $\mathrm{N}_{2}$ ) adsorbs on $\mathrm{BN}$ at the center of the hexagon at a distance of $3.3 \AA$, with an interaction energy of $3.90 \mathrm{mE}_{h}$. $E_{\mathrm{ad}}=3.55 \mathrm{mE}_{h}$ was estimated for $\mathrm{N}_{2}$ on graphite from heat of adsorption measurements at low coverage. ${ }^{54}$

At long range, BS effects are much less important. This is in fact not surprising: the two-electron excitations which contribute to the correlation energy are far more complex when the two systems are in close proximity, and require for their faithful description a rich variational freedom of the virtual space, which becomes less essential when the mutual interaction takes place at larger distances. After fitting the long-range part $(6.5 \AA<R<10.5 \AA)$ of the present HF + MP2 data with an $-\alpha R^{-4}$ curve, the following values (a.u.) are obtained for the best-fit $\alpha$ parameters for the different BSs: BSA: 7.24; BSB: 6.89; BSC: 7.54; BSD: 8.16 a.u. These are reasonably similar among themselves, slightly increasing when high angular momentum polarization functions (which are essential for a better description of the polarizability) are included in the basis. 


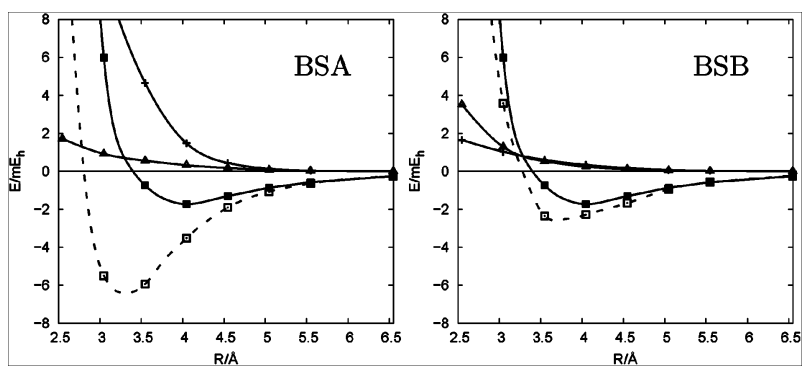

Fig. 3 HF BSSE (pluses) and MP2 BSSE (triangles) contributions to the final HF + MP2 (filled squares) interaction energy curve for BSA and BSB; the curves not corrected for the BSSE are also reported for comparison (open squares and dashed line).

Furthermore, these results are close to the $C_{4}^{\perp}$ values obtained via explicit evaluation of the polarizabilities (see Table 3). Important to note that, since the MP2 method treats dispersion at the uncoupled HF level, the LMP2 results should be closer to the orbital-unrelaxed SOS technique. This is indeed reproduced in our calculations. At the same time, inclusion of the orbital relaxation, vital for the zz-component of the static polarizability in this system (see Table 2), becomes substantially less important for the $C_{4}$ coefficients, which makes the SOS results just slightly surpass those of CPKS (see Table 3). This supports our observation of the relatively high accuracy of the LMP2 method in obtaining the $C_{4}$ coefficients for this system. Besides, on this basis we generally expect the MP2 description of molecular adsorption on the $\mathrm{BN}$ surface to be reasonably good.

The SOS curve reported in Fig. 2 is in fact in excellent agreement with the $\mathrm{HF}+\mathrm{MP} 2$ (BSA, BSB) data at long range; this may be partly due to compensation of errors, because the polarizabilities used for the slab should be slightly overestimated due to the "gap problem" (see section IV-B). For $R<4 \AA$, the SOS curve is always below the HF + MP2 data since it doesn't include any repulsive term. In an intermediate region ( $4 \AA<R<6.5 \AA$ ), however, the $\mathrm{HF}+\mathrm{MP} 2$ data reveal an additional attractive contribution, not accounted for by the $C_{6}$ term. It is natural to interpret this fact as due to the non-negligible importance, at these distances, of other dispersive interactions: dipole-quadrupole $\left(C_{8}\right)$, quadrupolequadrupole $\left(C_{10}\right)$, etc. Since our techniques do not yet allow us to calculate the corresponding coefficients, we have obtained an estimate of the " $C_{8}$ " contribution by assuming that the $C_{8} / C_{6}$ ratio in the present case is the same as the one semi-empirically evaluated for the argon/BN interaction. ${ }^{36}$ The dashed curve in Fig. 2, which corresponds to adding to the $C_{6}$ term the $C_{8}$ one so obtained, shows that our interpretation is tenable.

A few words on the BSSE correction: as already pointed out, all results are BSSE corrected through the classical counterpoise (CP) technique. As expected, the HF BSSE is quite large for BSA and rather small for BSB; at the MP2 level, instead, the BSSE is always small, which is a common feature of the local correlation methods. ${ }^{55}$ Fig. 3 reports the separate BSSE contributions to the $\mathrm{HF}+\mathrm{MP} 2$ interaction energy obtained and shows how important this correction is for poor basis sets.

\section{Conclusions}

In this paper we have addressed the problem of obtaining ab initio estimates of the coefficients which describe dispersive interactions between subunits which may be part of extended periodic systems. This is an important issue, because otherwise one should adopt for this purpose a semi-empirical approach, or use high-level theoretical schemes which are extremely costly or totally unfeasible in those cases.

The interaction of a nitrogen molecule with a thin film of hexagonal $\mathrm{BN}$ has been chosen as a case study for identifying some of the problems involved in the determination of the leading long-range term $\left(C_{6}\right)$, based on the independent analysis of the response properties of the two interacting systems (one of which two-dimensionally periodic), and for proposing techniques for their solution. Compared to the previous van der Waals results obtained between $\mathrm{N}_{2}$ and hexagonal $\mathrm{BN}$ in ref. 8, the transverse polarizability of h-BN surface has been here improved by CPKS calculations taking into account very large relaxation effects, which results in slightly lower $C_{4}$ values.

The simplicity of this problem has also permitted us to apply to it an ab initio post-HF (MP2) approach which adequately describes dispersive interactions; the newly implemented periodic code CRYSCOR has been used for this purpose, along with a supercell model. The results so obtained nicely confirm the accuracy of the estimate of the $C_{6}$ term owing to the other approach.

Prospective work includes the use in the techniques here described of one-electron Hamiltonians containing a non-local exchange term, and their application to other systems, in order to validate them more generally. We also envisage their extension to the determination of other dispersive terms which become important at intermediate range, such as the dipole-quadrupole $\left(C_{8}\right)$ one.

\section{References}

$1 \mathrm{X}$. Wu, M. C. Vargas, S. Nayak, V. Lotrich and G. Scoles, J. Chem. Phys., 2001, 115, 8748.

2 Q. Wu and W. Yang, J. Chem. Phys., 2002, 116, 515-524.

3 U. Zimmerli, M. Parrinello and P. Koumotsakos, J. Chem. Phys., 2004, 120, 2693.

4 S. Grimme, J. Comput. Chem., 2006, 27, 1787-1799.

5 P. Jurecka, J. Cerny, P. Hobza and D. R. Salahub, J. Comput. Chem., 2007, 28, 555-569.

6 C. Cohen-Tannoudji, B. Diu and F. Laloe, Mécanique quantique, Collection Enseignement des sciences, Hermann, Paris, 1973.

7 B. Civalleri, C. M. Zicovich-Wilson, L. Valenzano and P. Ugliengo, Cryst. Eng. Comm., 2008, 10, 405.

8 I. Baraille, M. Rérat and P. Mora, Phys. Rev. B: Condens. Matter Mater. Phys., 2006, 73, 75410.

9 C. Pisani, L. Maschio, S. Casassa, M. Halo, M. Schütz and D. Usvyat, J. Comput. Chem., 2008, 29, 2113.

10 CRYSCOR09 User's Manual. A. Erba and M. Halo; Università di Torino, Torino; http://www.cryscor.unito.it.

11 CRYSTAL09 User's Manual. R. Dovesi, V. R. Saunders, C. Roetti, R. Orlando, C. M. Zicovich-Wilson, F. Pascale, K. Doll, N. M. Harrison, B. Civalleri, I. J. Bush, P. D'Arco and M. Llunell, Università di Torino, Torino, 2009; http://www. crystal.unito.it.

12 Y. H. Kim, K. J. Chang and S. G. Louie, Phys. Rev. B: Condens. Matter, 2001, 63, 205408.

13 A. Rubio, J. L. Corkill and M. Cohen, Phys. Rev. B: Condens. Matter, 1994, 49, 5081 
14 X. Blase, A. Rubio, S. G. Louie and M. Cohen, Europhys. Lett., 1994, 28, 335

15 N. G. Chopra, R. J. Luyken, K. Cherrey, V. H. Crespi, M. Cohen, S. G. Louie and A. Z. 1, Science, 1995, 269, 966.

16 H. B. G. Casimir and D. Polder, Phys. Rev., 1948, 73, 360.

17 P. Pulay, Chem. Phys. Lett., 1983, 100, 151.

18 P. Pulay, S. Saebø and W. Meyer, J. Chem. Phys., 1984, 81, 1901.

19 S. Saebø and P. Pulay, Chem. Phys. Lett., 1985, 113, 13.

20 A. J. Stone, The Theory of Intermolecular Forces, Clarendon Press, Oxford, 1996.

21 M. Rérat, M. Mérawa and C. Pouchan, Phys. Rev. A: At., Mol., Opt. Phys., 1992, 45, 6263.

22 M. Rérat and B. Bussery-Honvault, Mol. Phys., 2003, 101, 373.

23 A. Hesselmann, G. Jansen and M. Schütz, J. Chem. Phys., 2005, 122, 014103.

24 A. J. Misquitta, R. Podeszwa, B. Jeziorski and K. Szalewicz, J. Chem. Phys., 2005, 123, 214103.

25 D. Ayma, J. P. Campillo, M. Rérat and M. Causà, J. Comput. Chem., 1997, 18, 1253.

26 P. Otto, Phys. Rev. B: Condens. Matter, 1992, 45, 10876.

27 M. Rérat, M. Ferrero, E. Amzallag, I. Baraille and R. Dovesi, J. Phys.: Conf. Ser., 2008, 117, 12023.

28 R. Resta and K. Kunc, Phys. Rev. B, 1986, 34, 7146.

29 C. Darrigan, M. Rérat, G. Mallia and R. Dovesi, J. Comput. Chem., 2003, 24, 1305.

30 M. Rérat and R. Dovesi, International Conference of Computational Methods in Sciences and Engineering, 2004, 1, 771.

31 M. Ferrero, M. Rérat, R. Orlando and R. Dovesi, J. Comput. Chem., 2008, 29, 1450.

32 M. Ferrero, M. Rérat, R. Orlando, R. Dovesi and I. J. Bush, J. Phys.: Conf. Ser., 2008, 117, 12016.

33 L. X. Benedict, S. G. Louie and M. L. Cohen, Phys. Rev. B: Condens. Matter, 1995, 52, 8541.

34 C. Kittel, Introduction to Solid State Physics, John Wiley \& Sons, Inc., New York, 5th edn, 1976.
35 E. N. Brothers, G. E. Scuseria and K. N. Kudin, J. Phys. Chem. B, 2006, 110, 12860.

36 G. Curthoys and P. Elkington, J. Phys. Chem., 1967, 71, 1477.

37 J. Langlet, J. Caillet, J. Bergés and P. Reinhardt, J. Chem. Phys., $2003,118,6157$.

38 S. Boys and F. Bernardi, Mol. Phys., 1970, 19, 553.

39 A. D. Becke, J. Chem. Phys., 1993, 98, 5648.

40 C. Lee, W. Yang and R. G. Parr, Phys. Rev. B, 1988, 37, 785.

41 J. T. H. Dunning, J. Chem. Phys., 1989, 90, 1007.

42 A. Grüneich and B. Heß, Theor. Chem. Acc., 1998, 100, 253.

43 B. Huron and P. Rancurel, Chem. Phys. Lett., 1972, 13, 515.

44 B. Huron, J. P. Malrieu and P. Rancurel, J. Chem. Phys., 1973, 58.

45 A. J. Sadlej, Collect. Czech. Chem. Commun., 1988, 53, 1995.

46 A. J. Sadlej, Theor. Chem. Acc., 1992, 81, 45.

47 A. J. Sadlej, Theor. Chim. Acta, 1992, 81, 339.

48 L. Museur, E. Feldbach and A. Kanaev, Phys. Rev. B: Condens. Matter Mater. Phys., 2008, 78, 155204.

49 B. Arnaud, S. Lebègue, P. Rabiller and M. Alouani, Phys. Rev. Lett., 2006, 96, 026402.

50 M. Schütz, D. Usvyat, M. Lorenz, C. Pisani, L. Maschio, S. Casassa and M. Halo, in Accurate Condensed-Phase Quantum Chemistry, ed. F. R. Manby, CRC Press, Taylor and Francis, NY, 2010, pp. 29-55.

51 L. Maschio, D. Usvyat, M. Schütz and B. Civalleri, J. Chem. Phys., 2010, 132, 134706.

52 D. Usvyat, L. Maschio, C. Pisani and M. Schütz, Z. Phys. Chem., 2010, 224, 441.

53 S. Rumyantsev, M. Levinshtein, A. Jackson, S. Mohammad, G. Harris, M. Spencer and M. Shur, Properties of Advanced Semiconductor Materials GaN, AlN, InN, BN, SiC, SiGe, John Wiley \& Sons, New York, 2001.

54 N. Avgul and A. Kiselev, Chemistry and Physics of Carbon, Dekker, New York, vol. 6, 1970.

55 M. Schütz, G. Rauhut and H. J. Werner, J. Phys. Chem. A, 1998, 102, 5997. 